\title{
ETV4 is a useful marker for the diagnosis of CIC-rearranged undifferentiated round-cell sarcomas: a study of 127 cases including mimicking lesions
}

Sophie Le Guellec ${ }^{1,2}$, Valérie Velasco ${ }^{3}$, Gaëlle Pérot ${ }^{2,3}$, Sarah Watson ${ }^{4}$, Franck Tirode ${ }^{4}$ and Jean-Michel Coindre ${ }^{2,3,5}$

${ }^{1}$ Department of Pathology, Institut Claudius Regaud, Institut Universitaire du Cancer Toulouse-Oncopole, Toulouse, France; ${ }^{2}$ INSERM U916, Institut Bergonié, Bordeaux, France; ${ }^{3}$ Department of Biopathology, Institut Bergonié, Bordeaux, France; ${ }^{4}$ INSERM U830, Laboratory of Genetics and Biology of Cancer, Institut Curie, Paris, France and ${ }^{5}$ Université de Bordeaux, Bordeaux, France

Subsets of primitive round-cell sarcomas remain difficult to diagnose and classify. Among these is a rare roundcell sarcoma that harbors a $C I C$ gene rearrangement known as $C I C$-rearranged undifferentiated round-cell sarcoma, which is most commonly fused to the DUX4 gene. Owing to its aggressive clinical behavior and potential therapeutic implications, accurate identification of this novel soft tissue sarcoma is necessary. Definitive diagnosis requires molecular confirmation, but only a few centers are as yet able to perform this test. Several studies have shown that PEA3 subfamily genes, notably ETV4 (belonging to the family of ETS transcription factors), are upregulated in $\mathrm{CIC}$-rearranged undifferentiated round-cell sarcomas. We performed a detailed immunohistochemical analysis to investigate ETV4 expression in CIC-rearranged undifferentiated round-cell sarcomas and their potential mimics (especially Ewing sarcomas). The study cohort included 17 cases of CIC-rearranged undifferentiated round-cell sarcomas, and 110 tumors that morphologically mimic $\mathrm{CIC}$-rearranged undifferentiated round-cell sarcomas: 43 Ewing sarcomas, 25 alveolar rhabdomyosarcomas, 20 poorly differentiated round-cell synovial sarcomas, 10 desmoplastic round-cell tumors, 5 BCOR-CCNB3 sarcomas, 5 lymphoblastic lymphomas, and 2 rhabdoid tumors. All CIC-rearranged undifferentiated round-cell sarcomas (on core needle biopsies and open biopsies) were ETV4-positive with a strong diffuse nuclear pattern. Among the other 110 tumors, only six cases (four Ewing sarcomas, one alveolar rhabdomyosarcoma, and one desmoplastic round-cell tumor) showed focal ( $<5 \%$ of tumor cells) and very weak nuclear expression of ETV4; all other tumors were completely negative for ETV4. We conclude that systematic immunohistochemical analysis of ETV4 makes it possible to diagnose undifferentiated round-cell sarcomas (with no molecular markers for sarcoma-associated translocation) such as $\mathrm{CIC}$-rearranged undifferentiated round-cell sarcoma. Modern Pathology (2016) 29, 1523-1531; doi:10.1038/modpathol.2016.155; published online 26 August 2016

$C I C$-rearranged undifferentiated round-cell sarcomas constitute a rare new entity that affects children and young adults. They have primitive small round-cell morphology and rearrangement of the CIC gene, which is located on chromosome 19q13.2 and is

Correspondence: Dr S Le Guellec, MD, Department of Pathology, Institut Claudius Regaud, Institut Universitaire du Cancer Toulouse-Oncopole, 1 avenue Irène Joliot-Curie, 31059 Toulouse Cedex 9, France.

E-mail: LeGuellec.sophie@iuct-oncopole.fr

Received 28 April 2016; revised 17 July 2016; accepted 18 July 2016; published online 26 August 2016 commonly fused to the DUX4 gene on chromosome $4 \mathrm{q} 35.2$ or $10 \mathrm{q} 26.3 .{ }^{1-3}$ Although alternative fusion partners such as the $\mathrm{FOXO} 4$ gene have recently been identified in some tumors, ${ }^{4,5}$ the partner genes in some CIC-rearranged undifferentiated round-cell sarcomas remain unknown. ${ }^{6}$ Owing to its aggressive clinical behavior, high propensity to metastasize, and quick-developing chemoresistance, ${ }^{6,7}$ recognition and accurate identification of this 'stand-alone' category of sarcoma is crucial. However, the small round cell's undifferentiated morphology (often mimicking Ewing sarcoma) and the lack of any lineage-specific markers can be challenging for 
diagnosis and classification. Moreover, definitive diagnosis of CIC-rearranged undifferentiated roundcell sarcoma requires cytogenetic or molecular confirmation especially by fluorescent in situ hybridization (FISH; showing CIC gene rearrangement), but such technology is currently available only at a few sarcoma centers. In 2006, Kawamura-Saito et al ${ }^{2}$ showed that fusion of DUX4 to the CIC sequence provides strong transcriptional activity, suggesting that expression of its downstream targets might be deregulated. Gene expression analysis identified the ETS family genes (ETV5 and ETV1) as potential targets for the gene products of CIC-DUX4. Recently, Specht et $a l^{8}$ confirmed these data by showing that CIC-DUX4 fusion upregulates the ETV4 gene, a PEA3 subfamily gene that belongs to the family of ETS transcription factors. Moreover, Specht et al ${ }^{8}$ argue that WT1 (N-terminal) immunoreactivity in CICrearranged undifferentiated round-cell sarcomas may serve as a useful marker in the differential diagnosis of Ewing sarcoma.

In this study, we evaluated the potential role of ETV4 and WT1 (N- and C-terminal) immunohistochemical expression in routine practice for the positive and differential diagnosis of CIC-rearranged undifferentiated round-cell sarcomas from other blue small round-cell sarcomas.

\section{Materials and methods}

\section{Case and Sample Selection}

Ethics approval from the appropriate committees was obtained. Formalin-fixed paraffin-embedded specimens accrued between August 2014 and November 2015 were retrieved from the pathology archives of Institut Bergonié (Bordeaux, France). The vast majority of cases were consult cases. All tissue samples were obtained via image-guided core needle biopsy or open biopsy. The study cohort included 17 cases of CIC-rearranged undifferentiated round-cell sarcomas and 105 non-CIC-rearranged undifferentiated round-cell sarcomas. For the CIC-rearranged undifferentiated round-cell sarcomas cohort, the following clinical data were collected: gender, age at diagnosis, and location and size of the tumor. The diagnosis of CIC-rearranged undifferentiated roundcell sarcoma was established by using FISH to verify the presence of CIC gene rearrangement. The heterogeneous group of non-CIC-rearranged undifferentiated round-cell sarcomas contained four main subgroups: 43 Ewing sarcomas, which harbor the pathognomonic canonical EWSR1-ETS translocation detected by reverse-transcriptase polymerase chain reaction (RT-PCR) and sequencing analysis or by EWSR1 FISH break-apart (data not shown); 25 alveolar rhabdomyosarcomas, with the characteristic FOXO1 gene rearrangement (FISH break-apart; data not shown), or PAX3-FOXO1 or PAX7-FOXO1 translocation (RT-PCR and sequencing analysis; data not shown); 20 poorly differentiated round-cell synovial sarcomas that have a specific SS18 gene rearrangement (FISH break-apart; data not shown); 10 desmoplastic round-cell tumors for which WT1-EWSR1 fusion gene was detected by RT-PCR (data not shown), 5 BCOR-CCNB3 sarcomas for which BCORCCNB3 fusion gene was detected by RT-PCR (data not shown), 5 lymphoblastic lymphomas and 2 rhabdoid tumors (biallelic deletion of INI1 gene). Hematoxylin and eosin-stained slides and previously performed immunohistochemical stains were reviewed in all the cases.

\section{Immunohistochemistry}

Core needle biopsy or open biopsy samples of the 127 cases were deparaffinized in xylene, hydrated in alcohol, and baked in a microwave (30 min in Trisbuffer, pH 9). Endogenous peroxidase was blocked. Tissue sections of the 127 samples were incubated at $4{ }^{\circ} \mathrm{C}$ overnight $(20 \mathrm{~h})$ using a monoclonal anti-ETV4 (clone 16, dilution 1:20; Santa Cruz Biotechnology, Santa Cruz, CA, USA). Tissue sections were washed with Tris-buffered saline and Tween 20 (TBST), then incubated for $40 \mathrm{~min}$ with 'Envision/DAB+ kit' (Dako, Glostrup, Denmark), washed with TBST, and incubated for $10 \mathrm{~min}$ with diamino-benzidine solution (Dako). Colon adenocarcinoma (nuclear staining) was used as positive control for ETV4.

In all available cases of $C I C$-rearranged undifferentiated round-cell sarcomas, six supplementary antibodies were used, and staining was performed on the Benchmark ultra-automated stainer (Ventana) using diamino-benzidine as chromogen: anti-WT1 (N-terminal; 6F-H2, pre-diluted, Dako), anti-WT1 (C-terminal; 1:100; Zytomed Systems, Berlin, Germany), anti-CD99 monoclonal antibody (1:100; clone 12E7, Dako), anti-Fli1 (1:300; C19, Santa Cruz Biotechnology), anti-ERG (1:100; EP111, Epitomics, Burlingame, CA, USA), and anti-cyclin B3 (1:200; Sigma Aldrich, St Louis, MO, USA). In 70 nonCIC-rearranged undifferentiated round-cell sarcomas, (25 Ewing sarcomas, 20 alveolar rhabdomyosarcomas, 20 poorly differentiated round-cell synovial sarcomas, 2 desmoplastic round-cell tumors, 2 BCOR-CCNB3 sarcomas, and 1 rhabdoid tumor), antibody against WT1 (C-terminal) was used. Finally, antibody against WT1 (N-terminal) was tested in 10 Ewing sarcomas. Immunohistochemical expression was quantitatively scored according to the percentage of positive cells. Location of staining was specified as nuclear or cytoplasmic. The intensity of staining was graded as weak (1+), moderate $(2+)$, or strong (3+). A positive control was tested on each immunohistochemical run.

\section{Fluorescence In situ Hybridization}

FISH assay was performed using the Histology FISH accessory kit (Dako) according to the manufacturer's 
instructions. CIC probe was prepared with BAC clones labeled using a Nick translation reagent kit according to the manufacturer's instructions (Abbott). For the CIC gene 5' part, $250 \mathrm{ng}$ of RP11343B1 and $250 \mathrm{ng}$ of RP11-688M4 BAC were labeled with spectrum orange-dUTP, and for the CIC gene $3^{\prime}$ part, $250 \mathrm{ng}$ of RP11-778C1 and 250 ng of RP11780011 BAC were labeled using spectrum greendUTP (Abbott). Labeled BAC DNA were then precipitated with $25 \mu \mathrm{g}$ of human Cot-1 DNA and resuspended in hybridization buffer $(50 \%$ formamide, $2 \times$ SSC pH 6.8, $1 \times$ Denhardt, $1 \%$ SDS, $40 \mathrm{mM}$ $\mathrm{NaH}_{2} \mathrm{PO}_{4} \mathrm{pH} 7,10 \%$ Dextran sulfate). One hundred nuclei were counted in each case under a Nikon Eclipse 80i fluorescent microscope with appropriate filters and a positive score was interpreted when at least $20 \%$ of the nuclei showed a break-apart signal. Nuclei with an incomplete set of signals were omitted from the score. Pictures were captured using a Hamamatsu C4742-95 CCD camera and analyzed using Genikon software (Alphelys, France).

\section{Results}

Clinicopathological and Immunohistochemical Characteristics of CIC-rearranged Undifferentiated Round-cell Sarcomas

Patient characteristics of CIC-rearranged undifferentiated round-cell sarcomas are presented in Table 1. The tumors were from 11 male and 6 female patients $(\mathrm{M} / \mathrm{F}$ sex ratio $=1.8)$. The mean and median age at diagnosis was 32 years and 24 years, respectively (range 12-83). Eight patients (54\%) were younger than 25 years. Eleven cases $(74 \%)$ arose from soft tissue and 4 cases $(26 \%)$ were visceral (one stomach, one colon, one kidney, and one lung). Fourteen cases (94\%) were deeply located. Tumors ranged from 2.5 to $15.0 \mathrm{~cm}$ in size $($ mean $=8.2)$.

\section{Histological Findings of CIC-rearranged Undifferentiated Round-cell Sarcomas}

Histologically, CIC-rearranged undifferentiated roundcell sarcoma have an undifferentiated small round blue cell tumor appearance and tend to grow in a lobular architectural configuration (Figure 1a). At lower magnification, they demonstrate large, localized areas of necrosis (Figures $1 \mathrm{~b}$ and $\mathrm{c}$ ) with viable cells forming perivascular cuffs within the necrotic areas (Figures $1 \mathrm{~b}$ and c). Geographic tumor necrosis was prominent in the majority of cases $(66 \%$ of cases, $n=10$ ). At higher magnification, tumor cells contained medium-sized round to vesicular nuclei and clear or pale eosinophilic cytoplasm, which was typically more abundant than in Ewing sarcoma (Figures 1c and d). Although no pleomorphic cells were visible, these tumors showed a higher degree of heterogeneity in nuclear size and shape compared with the rather monomorphic aspect seen in Ewing sarcoma. Mitotic counts ranged from 11-125 per 10 high-power fields (median count of 39). In small foci, the cells were arranged in a vague storiform pattern (Figure 1e). Patchy myxoid or edematous stromal change was sometimes seen (Figure 1f).

\section{Immunohistochemical Findings and Molecular Characteristics of $C I C$-rearranged Undifferentiated Round-cell Sarcomas}

The results of the immunohistochemical analysis are summarized in Table 2. All CIC-rearranged undifferentiated round-cell sarcoma cases tested were positive for ETV4 $(17 / 17 ; 100 \%)$, displaying a diffuse nuclear pattern of staining with strong intensity (3+; Figures 2a and b). Nuclear WT1 (C-terminal) staining was seen in 9/12 (75\%) CIC-rearranged undifferentiated round-cell sarcomas (Figures 2c and d). Cytoplasmic WT1 (N-terminal) was positive

Table 1 Clinical features of $C I C$-rearranged round-cell sarcomas

\begin{tabular}{|c|c|c|c|c|c|c|c|}
\hline Case & Age (years) & Gender & Location & Depth & Size $(\mathrm{cm})$ & Necrosis & Number of mitoses/10 HPF \\
\hline 1 & 14 & $\mathrm{M}$ & Colon & Deep & 14 & No & 25 \\
\hline 2 & 19 & $\mathrm{M}$ & Spine & Deep & 8 & Yes & 46 \\
\hline 3 & 47 & $\mathrm{M}$ & Spine & Deep & 4.5 & No & 22 \\
\hline 4 & 42 & $\mathrm{~F}$ & Thigh & $\mathrm{S}$ & NA & Yes & 20 \\
\hline 5 & 12 & $\mathrm{~F}$ & Back & Deep & 5 & Yes & 68 \\
\hline 6 & 24 & $\mathrm{~F}$ & Stomach & Deep & 5 & No & NA \\
\hline 7 & 20 & M & Head/neck & Deep & 5.5 & Yes & 58 \\
\hline 8 & 43 & $\mathrm{M}$ & Chest wall & Deep & 2.5 & Yes & 25 \\
\hline 9 & 53 & $\mathrm{~F}$ & Lung & Deep & 11 & Yes & 22 \\
\hline 10 & 83 & $\mathrm{~F}$ & Kidney & Deep & 14.5 & Yes & 20 \\
\hline 11 & 20 & $\mathrm{~F}$ & Pleural & Deep & NA & Yes & 20 \\
\hline 12 & 18 & M & Chest wall & Deep & 15 & Yes & 11 \\
\hline 13 & 26 & $\mathrm{M}$ & Thigh & Deep & NA & No & NA \\
\hline 14 & 47 & $\mathrm{M}$ & IVC & Deep & 5.5 & No & 125 \\
\hline 15 & 18 & $\mathrm{M}$ & Calf & Deep & NA & Yes & 43 \\
\hline 16 & 17 & M & Axillary & Deep & 3.2 & Yes & 30 \\
\hline 17 & 57 & $\mathrm{M}$ & Retro peritoneal & Deep & NA & Yes & 11 \\
\hline
\end{tabular}

Abbreviations: F, female; IVC, inferior vena cava; M, male; NA, not available; S, superficial. 

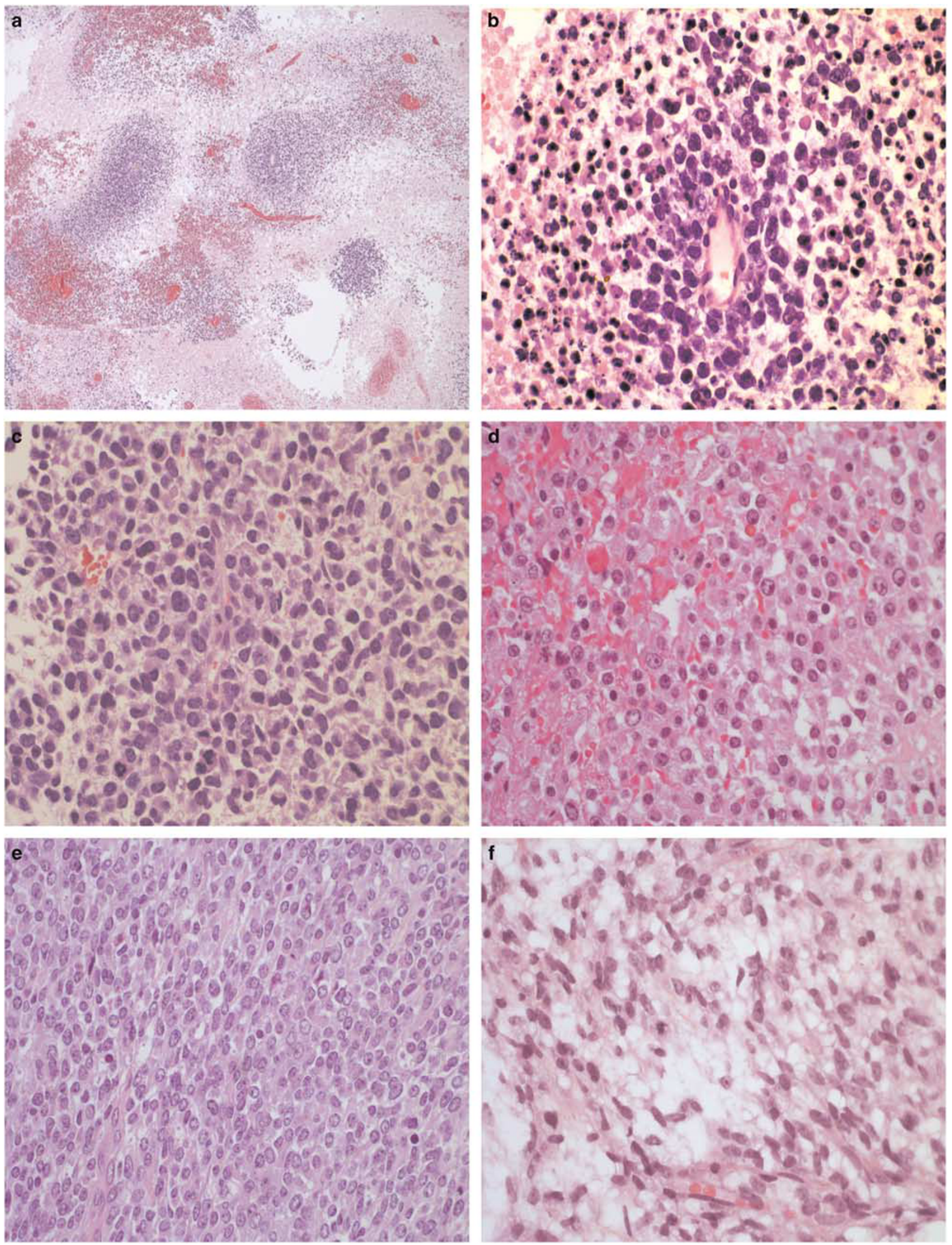
in 14 cases (14/17), and in one case, a combined nuclear and cytoplasmic pattern of staining was observed (1/17). Positive nuclear ERG was observed in only 1 case $(1 / 8 ; 12 \%)$ displaying focal $(10 \%$ of cells) and weak intensity (1+). Positive nuclear FLI1 was seen in 2 cases $(2 / 8 ; 25 \%)$ with only focal $(5 \%$ of cells) and weak intensity (1+). All CIC-rearranged undifferentiated round-cell sarcomas were negative for cyclin B3 (0/17). Seven cases stained for CD99 $(7 / 15)$ with membranous staining seen in 4 cases (Figure 2e) and only patchy weak staining in the other 3 cases. All cases (17/17) of CIC-rearranged undifferentiated round-cell sarcomas showed a CIC rearrangement with FISH (Figure 2f).

\section{Immunohistochemical Expression in Other Tumors}

Among other 110 tumors, only 6 cases (5\%; 4 Ewing sarcomas, 1 alveolar rhabdomyosarcoma, and 1 desmoplastic round-cell tumor) showed focal ( $<5 \%$ of tumors cells) and very weak ETV4 nuclear expression (1+). ETV4 expression was completely absent in all other 39 Ewing sarcomas (Figures 3a and b), all other 24 alveolar rhabdomyosarcomas (Figures 3c and d), the 20 poorly differentiated round-cell synovial sarcomas (Figures $3 e$ and f), all other 9 desmoplastic round-cell tumors, the 5 BCORCCNB3 sarcomas, 5 lymphoblastic lymphomas, and in the 2 rhabdoid tumors. Among the 70 non-CICrearranged undifferentiated round-cell sarcomas tested, 7 (10\%) were positive for WT1 (C-terminal), displaying nuclear diffuse ( $>50 \%$ ) strong $(3+)$ intensity in 3 cases (1 alveolar rhabdomyosarcoma and 2 desmoplastic round-cell tumors) and in 4 cases (3 Ewing sarcomas and 1 alveolar rhabdomyosarcoma) with focal $(<50 \%)$ nuclear strong intensity. Finally, 3 Ewing sarcomas displayed 'dot-like' focal positivity for WT1 (N-terminal) and 7 were negative.

\section{Discussion}

CIC-rearranged undifferentiated round-cell sarcomas are recently described aggressive tumors in the soft tissues of children and young adults. ${ }^{2,3}$ The diagnosis of $C I C$-rearranged undifferentiated round-cell sarcoma is challenging not only owing to the partial overlap of morphological features, especially with Ewing sarcoma, but also with other small blue round-cell tumors such as poorly differentiated round-cell synovial sarcoma, alveolar rhabdomyosarcoma, and desmoplastic round-cell tumors. ${ }^{8,9}$ Moreover, definitive diagnosis is on the basis of cytogenetic or molecular analyses (presently available only at a few centers) for identification of the

Table 2 Immunohistochemical features of CIC-rearranged round-cell sarcomas

\begin{tabular}{|c|c|c|c|c|c|c|c|}
\hline Case & CD99 & $\begin{array}{l}\text { Cyclin B3 } \\
\text { (\%, intensity, } \\
\text { location) }\end{array}$ & $\begin{array}{l}\text { Fli1 } \\
\text { (\%, intensity, } \\
\text { location) }\end{array}$ & $\begin{array}{l}\text { ERG } \\
\text { (\%, intensity, } \\
\text { location) }\end{array}$ & $\begin{array}{c}\text { WT1 (N-TER) } \\
\text { (\%, intensity, } \\
\text { location) }\end{array}$ & $\begin{array}{c}\text { WT1 (C-TER) } \\
\text { (\%, intensity, } \\
\text { location) }\end{array}$ & $\begin{array}{c}\text { ETV4 } \\
(\%, \text { intensity, } \\
\text { location) }\end{array}$ \\
\hline 1 & Negative & Negative & $\mathrm{ND}$ & ND & Negative & ND & $100,3+, \mathrm{N}$ \\
\hline 2 & Focal & Negative & Negative & ND & $100,3+, \mathrm{C}$ & $100,3+, \mathrm{N}$ & $100,3+, \mathrm{N}$ \\
\hline 3 & Negative & Negative & ND & ND & Negative & Negative & $100,3+, \mathrm{N}$ \\
\hline 4 & Pos, m & Negative & ND & ND & $100,3+, \mathrm{C}$ & $100,3+, \mathrm{N}$ & $100,3+, \mathrm{N}$ \\
\hline 5 & Pos, m & Negative & ND & ND & $100,3+, \mathrm{C}$ & Negative & $80,3+, \mathrm{N}$ \\
\hline 6 & Negative & Negative & Negative & Negative & $100,3+, \mathrm{C}$ & $100,3+, \mathrm{N}$ & $100,3+, \mathrm{N}$ \\
\hline 7 & Negative & Negative & $5,1+, \mathrm{N}$ & Negative & $100,3+, \mathrm{C}+\mathrm{N}$ & $100,3+, \mathrm{N}$ & $100,3+, \mathrm{N}$ \\
\hline 8 & Negative & Negative & Negative & Negative & $100,3+, \mathrm{C}$ & Negative & $100,3+, \mathrm{N}$ \\
\hline 9 & Negative & Negative & ND & Negative & Negative & ND & $100,3+, \mathrm{N}$ \\
\hline 10 & ND & Negative & $5,1+, \mathrm{N}$ & Negative & $100,3+, \mathrm{C}$ & ND & $100,3+, \mathrm{N}$ \\
\hline 11 & Pos, m & Negative & ND & ND & $100,3+, \mathrm{C}$ & $100,3+, \mathrm{N}$ & $100,3+, \mathrm{N}$ \\
\hline 12 & Focal & Negative & ND & ND & $100,3+, \mathrm{C}$ & ND & $100,3+, \mathrm{N}$ \\
\hline 13 & ND & Negative & ND & Negative & $100,3+, \mathrm{C}$ & $100,3+, \mathrm{N}$ & $100,3+, \mathrm{N}$ \\
\hline 14 & Pos, m & Negative & Negative & $10,1+, \mathrm{N}$ & $100,3+, \mathrm{C}$ & $100,3+, \mathrm{N}$ & $100,3+, \mathrm{N}$ \\
\hline 15 & Negative & Negative & ND & ND & $100,3+, \mathrm{C}$ & ND & $100,3+, \mathrm{N}$ \\
\hline 16 & Focal & Negative & Negative & Negative & $100,3+, \mathrm{C}$ & $100,3+, \mathrm{N}$ & $80,3+, \mathrm{N}$ \\
\hline 17 & Negative & Negative & Negative & Negative & $100,3+, \mathrm{C}$ & $100,3+, \mathrm{N}$ & $100,3+, \mathrm{N}$ \\
\hline
\end{tabular}

Abbreviations: C, cytoplasmic; Pos, m, membranous positivity; N, nuclear; ND, not done.

Figure 1 Morphological features of CIC-rearranged undifferentiated round-cell sarcomas. (a) Open biopsy: tumor showing vague nodular growth with localized areas of necrosis and perivascular cell preservation (hematoxylin and eosin (H\&E) $\times 40$ ). (b) Open biopsy: tumor cells arranged in solid sheets surrounding vessel with areas of necrosis. (H\&E $\times 400)$. (c) Tumor composed of cells displaying less monomorphic morphology compared with Ewing sarcoma. The nuclei appear oval, round to more angulated with increased pleomorphism and distinct nucleoli $(\mathrm{H} \& \mathrm{E} \times 400)$. (d) Some tumors showing focal sheets of round cells with more abundant amphophilic cytoplasm, vesicular chromatin, and prominent nucleoli (H\&E $\times 400)$. (e and f) Rare tumors showing focal areas of spindle cells arranged in vague storiform pattern. Edematous and myxoid stroma is not uncommon $(\mathrm{H} \& \mathrm{E} \times 400)$. 

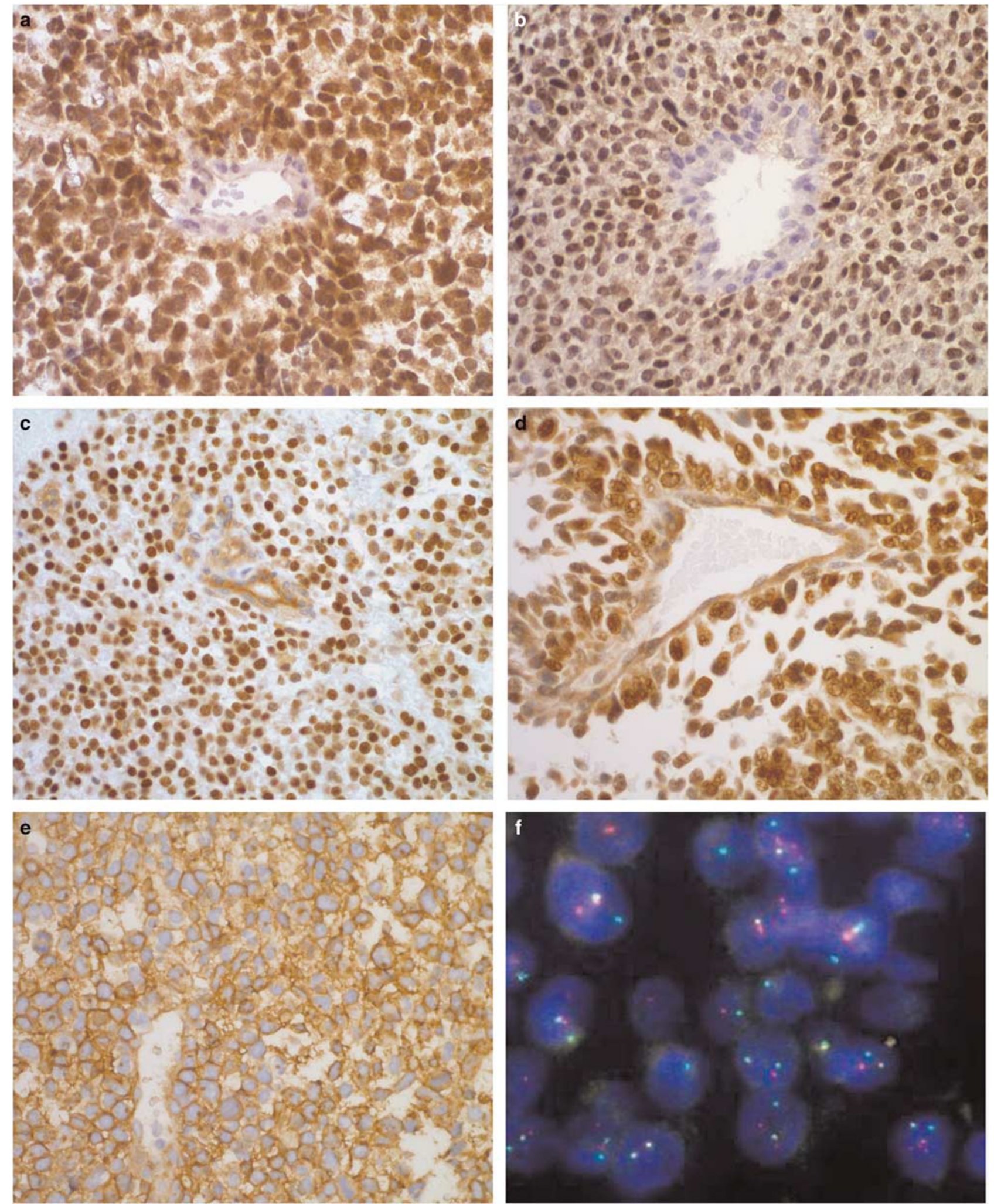

Figure 2 ETV4 expression in CIC-rearranged undifferentiated round-cell sarcomas. (a and b) Tumor cells showing diffuse and strong nuclear expression of ETV4 with negative endothelial cells (internal negative control; hematoxylin counterstain X400). (c and d) Tumor cells showing diffuse and strong nuclear expression of WT1 (C-terminal; hematoxylin counterstain X400). (e) CD99 immunostaining showing strong and diffuse membranous reactivity in CIC-rearranged undifferentiated round-cell sarcoma (hematoxylin counterstain $\times 400$ ). (f) Fluorescent in situ hybridization analysis in CIC-rearranged undifferentiated round-cell sarcoma: probes flanking the CIC locus (CIC gene 5' part labeled in orange and CIC gene 3' part labeled in green): a signal pattern consisting of one red/green fusion signal, one red signal, and a separate red signal indicates, respectively, one wild-type CIC allele and the second allele split owing to the (4;19)(q35;q13.1) rearrangement. 

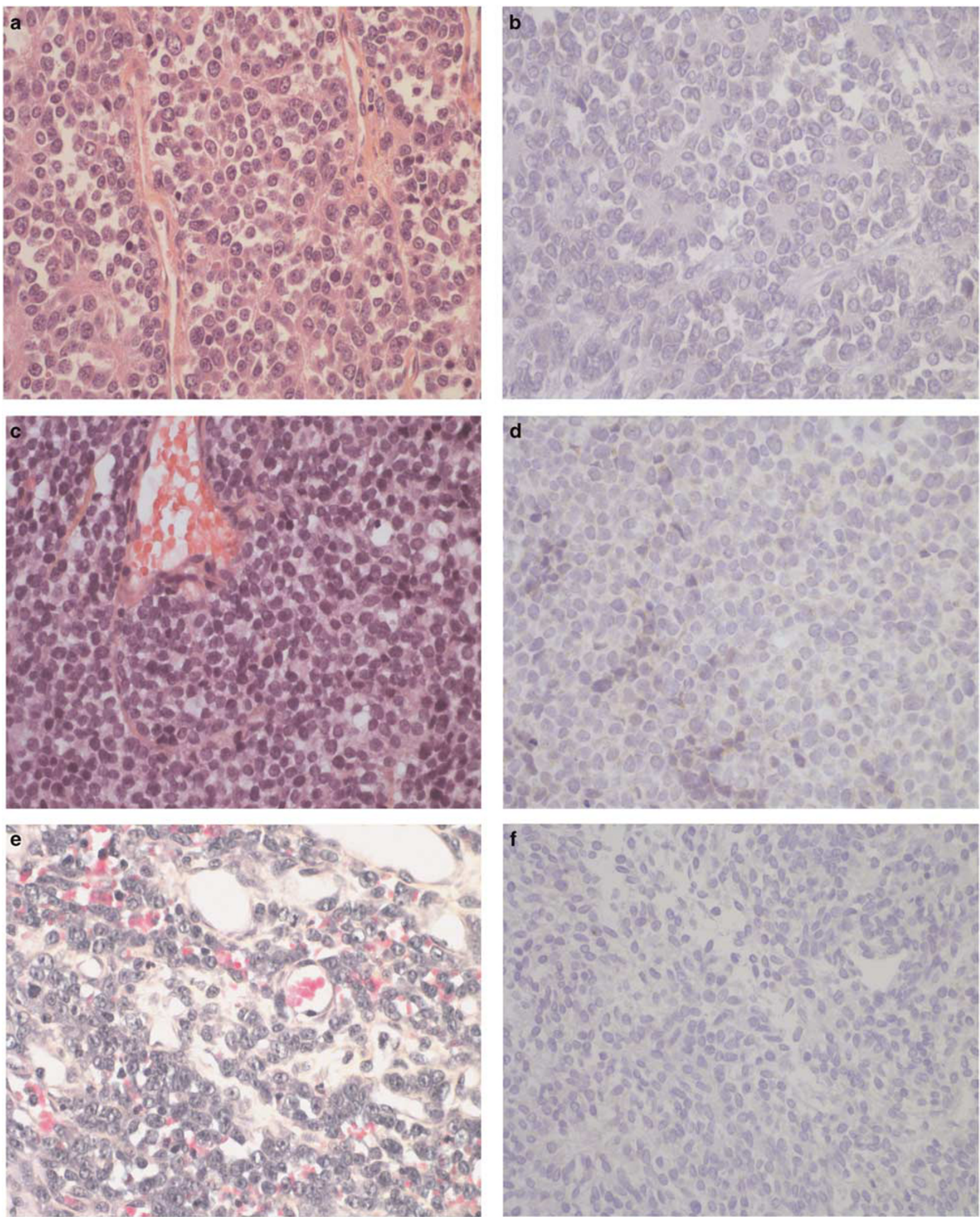

Figure 3 Histological and immunohistochemical characteristics of tumors mimicking CIC-rearranged undifferentiated round-cell sarcoma. (a and b) Ewing sarcoma. (a) Uniform small round cells with round nuclei containing fine chromatin and scanty eosinophilic cytoplasm. Of note, rosette formation was observed (hematoxylin counterstain $\times 400$ ). (b) Tumor cells showing no expression of ETV4. (c and d) Alveolar rhabdomyosarcoma, c, the solid variant of alveolar rhabdomyosarcoma displays sheet of undifferentiated round cells without fibrovascular septa. (d) No expression of ETV4 by tumor cells. (e and f) Poorly differentiated, round-cell synovial sarcoma. (e) Small round cells with nuclear crowding, hyperchromatic nuclei, and presence in places of prominent nucleoli. (f) Tumor cells showing no expression of ETV4. 
specific rearrangement. Although CIC-rearranged undifferentiated round-cell sarcomas are rare (about 60 cases reported in the literature), ${ }^{8,10}$ this entity represents up to two-thirds of EWSR1 rearrangementnegative round-cell sarcomas. ${ }^{6}$ In this study, we report 17 new cases of CIC-rearranged undifferentiated round-cell sarcomas with clinicopathologic data. We demonstrate the nuclear expression of ETV4 in these sarcomas and show that their potential morphological mimics do not express ETV4. Altogether, these data suggest that ETV4 expression is a useful tool for diagnosing CIC-rearranged undifferentiated roundcell sarcoma.

The first case of $t(4 ; 19)(q 35 ; q 13.1)$-associated sarcoma was reported in 1996 by Richkind et $a l^{1}$ in a 12-year-old boy who presented a large ankle soft tissue mass with concomitant lung metastases and died within 10 months. Ten years later, KawamuraSaito et $a l^{2}$ identified CIC (19q13) and DUX4 (4q35) genes resulting from $t(4 ; 19)(q 35 ; q 13.1)$ in two adult patients. Recently, DUX4L10 (located on chromosome 10q26.3), a D4Z4 repeat array similar to DUX4, was shown to be another fusion partner for $C I C .{ }^{6}$ In 2014, an alternative fusion partner to $C I C$, the FOXO4 gene, was identified in few tumors, and their clinicopathologic features were similar to those described in CIC-DUX4 round-cell sarcomas. ${ }^{4,5}$ Initially referred to as 'Ewing-like sarcoma'2 or 'atypical Ewing sarcoma', 3 CIC-rearranged undifferentiated round-cell sarcoma is currently considered as a pathological entity distinct from Ewing sarcoma. ${ }^{7,8}$ Although CIC-rearranged undifferentiated round-cell sarcoma currently represents a stand-alone category of sarcomas, the distinction between Ewing sarcoma and CIC-rearranged undifferentiated round-cell sarcoma on the basis of morphological and immunohistochemical features appears to be challenging. However, the clinical and pathological characteristics of the 60 reported cases in the literature showed important differences compared with Ewing sarcoma. The 17 new cases in this study show the same features as those described in the 60 cases reported in the literature. ${ }^{1-3,6,7-11}$ First of all, $C I C$-rearranged undifferentiated round-cell sarcomas mostly occur in young adults with a peak incidence in the third decade, whereas patients with Ewing sarcoma have a mean age at diagnosis of 15 years. ${ }^{12-13}$ Second, unlike Ewing sarcoma that often presents as a primary bone tumor, ${ }^{12}$ CIC-rearranged undifferentiated round-cell sarcomas are extraskeletal lesions with the majority occurring in the deep soft tissue, albeit, at a variety of visceral locations. From a morphological point of view, although both tumors show a monotonous and undifferentiated morphology, CIC-rearranged undifferentiated roundcell sarcoma show a greater degree of morphological heterogeneity with coarse chromatin, the presence of nucleoli, and more abundant cytoplasm. ${ }^{6,8}$ In addition, CIC-rearranged undifferentiated round-cell sarcomas often have a myxoid matrix, which is usually not seen in Ewing sarcoma. Immunohistochemically, staining for CD99 in CIC-rearranged undifferentiated round-cell sarcomas is variable, usually showing a membranous pattern, but often being weak in intensity and focal in distribution when compared with Ewing sarcoma, which shows diffuse strong membranous reactivity. ${ }^{6,8,9,14}$ Although these differences are real, they remain subtle, so additional robust and convenient marker(s) would be useful in daily practice to better identify $C I C$-rearranged undifferentiated round-cell sarcoma. Indeed, owing to their aggressive clinical behavior and resultant therapeutic implications, their accurate identification is important.

The CIC-DUX4 fusion chimeric protein acts as an aberrant transcription factor with transforming activity and strong transcriptional activation of downstream CIC targets. ${ }^{2}$ The CIC-DUX4 oncoprotein leads to upregulation of several ETS family genes (ETV4, ETV1, and ETV5) from the PEA3 subfamily (polyoma enhancer activator 3). ${ }^{8,13}$ Moreover, Specht et $a l^{8}$ showed that ETV4 gene expression level was not significantly elevated in Ewing sarcomas (with EWSR1-FLI1 or EWSR1-ERG translocation) compared with that in CIC-rearranged undifferentiated round-cell sarcomas. Accordingly, we observed ETV4 staining in all CIC-rearranged undifferentiated round-cell sarcomas with a diffuse nuclear pattern and strong intensity. In contrast, ETV4 immunohistochemistry was completely negative in 104/110 (95\%) of all small blue round-cell sarcomas tested. Four Ewing sarcomas showed ETV4 expression, but only focal ( $<5 \%$ of tumor cells) and very weak nuclear expression $(1+)$, which is totally different from the diffuse and strong immunohistochemical expression observed in CIC-rearranged undifferentiated round-cell sarcomas.

Specht et $a l^{8}$ recently reported that WT1 (N-terminal) expression may provide a useful clue in the diagnosis of CIC-rearranged undifferentiated round-cell sarcoma. In our study, 14 CIC-rearranged undifferentiated round-cell sarcomas expressed WT1 (N-terminal) exclusively with a cytoplasmic pattern $(13 / 14)$, and only one with a nuclear and cytoplasmic pattern, and three were totally negative. This result is different from that published by Specht et $a l^{8}$ who reported a combined nuclear and cytoplasmic pattern of staining in 15/20 CIC-rearranged tested, 4 with only nuclear staining, and 1 with only cytoplasmic reactivity. We tested the same anti-WT1 (N-terminal) antibody (6F-H2, Dako) and were unable to reproduce the results using the protocol described by Specht et $a l^{8}$ Interestingly, Yoshida et $a l^{10}$ reported that in a cohort of 20 CIC-rearranged undifferentiated round-cell sarcomas, they observed cytoplasmic and nuclear expression of WT1 (N-terminal) in 14 tumors (14/20, 70\%) with cytoplasmic WT1 (N-terminal) expression in all of them. However, they noted that cytoplasmic reactivity was often more prevalent and intense than nuclear staining, and that in certain tumors, only $20 \%$ of tumor cells were positive. Consequently, 
they conclude that WT1 (N-terminal) expression may have interpretative pitfalls. Therefore, this antibody seems to be difficult to analyze in this context, especially as the vast majority of these cases are consult material for which the pre-analytical variables of the samples (fixation and processing) are more or less homogeneous. On the other hand, WT1 (C-terminal) immunoreactivity in CIC-rearranged undifferentiated round-cell sarcomas may serve as a complementary marker of ETV4. Indeed, $75 \%$ of $C I C$-rearranged undifferentiated round-cell sarcomas tested (9/12) showed diffuse nuclear staining and strong intensity with WT1 (C-terminal), and among $C I C$-rearranged undifferentiated round-cell sarcomas mimics, only 10\% (7 cases) displayed nuclear staining, including 3 cases with strong $(3+)$ intensity and a diffuse pattern ( $>50 \%$ ).

In conclusion, ETV4 expression is a useful tool for diagnosing CIC-rearranged undifferentiated round-cell sarcoma. We show here that it is a sensitive and specific test to distinguish CIC-rearranged undifferentiated round-cell sarcoma from its morphological mimics. ETV4 expression analysis can also help in the differential diagnosis of other small round-cell tumors in the appropriate clinical, histological, and immunohistochemical context. WT1 (C-terminal), but not WT1 (N-terminal), may serve as a complementary marker for ETV4. When dealing morphologically with small blue round-cell tumors, which lack rearrangement of EWSR1, ETV4 expression allows cases to be selected for FISH analysis with a view to establishing the definitive diagnosis of CIC-rearranged undifferentiated round-cell sarcoma, especially on core needle biopsies.

\section{Acknowledgments}

We thank Dr C Laurent and Mme N Amara from Lymphopath consortium (French lymphoma network supported by the Institut National du Cancer) for sending five cases of lymphoblastic leukemia. We thank Dr Ravi Nookala from Institut Bergonié for medical writing assistance in English. The results of this study were presented in part at the United States and Canadian Academy of Pathology Meeting, Seattle, WA, USA, 12-18 March, 2016.

\section{Disclosure/conflict of interest}

The authors declare no conflict of interest.

\section{References}

1 Richkind KE, Romansky SG, Finklestein JZ. t(4;19)(q35; q13.1): a recurrent change in primitive mesenchymal tumors? Genes Chromosomes Cancer 2012;51:207-218.

2 Kawamura-Saito M, Yamazaki Y, Kaneko K, et al. Fusion between CIC and DUX4 up-regulates PEA3 family genes in Ewing-like sarcomas with $\mathrm{t}(4 ; 19)(\mathrm{q} 35 ; \mathrm{q} 13)$ translocation. Hum Mol Genet 2006;15:2125-2137.

3 Yoshimoto M, Graham C, Chilton-MacNeill S, et al. Detailed cytogenetic and array analysis of pediatric primitive sarcomas reveals a recurrent CIC-DUX4 fusion gene event. Cancer Genet Cytogenet 2009;195:1-11.

4 Solomon DA, Brohl AS, Khan J, et al. Clinicopathologic features of a second patient with Ewing-like sarcoma harboring CIC-FOXO4 gene fusion. Am J Surg Pathol 2014;38:1724-1725.

5 Sugita S, Arai Y, Tonooka A, et al. A novel CIC-FOXO4 gene fusion in undifferentiated small round cell sarcoma: a genetically distinct variant of Ewing-like sarcoma. Am J Surg Pathol 2014;38:1571-1576.

6 Italiano A, Sung YS, Zhang L, et al. High prevalence of CIC fusion with double-homeobox (DUX4) transcription factors in EWSR1-negative undifferentiated small blue round cell sarcomas. Genes Chromosomes Cancer 2012;51:207-218.

7 Choi EY, Thomas DG, McHugh JB, et al. Undifferentiated small round cell sarcoma with t(4;19)(q35;q13.1) CIC-DUX4 fusion: a novel highly aggressive soft tissue tumor with distinctive histopathology. Am J Surg Pathol 2013;37:1379-1386.

8 Specht K, Sung YS, Zhang L, et al. Distinct transcriptional signature and immunoprofile of CIC-DUX4 fusion-positive round cell tumors compared to EWSR1-rearranged Ewing sarcomas: further evidence toward distinct pathologic entities. Genes Chromosomes Cancer 2014;53:622-633.

9 Graham C, Chilton-MacNeill S, Zielenska M, et al. The CIC-DUX4 fusion transcript is present in a subgroup of pediatric primitive round cell sarcomas. Hum Pathol 2012;43:180-189.

10 Yoshida A, Goto K, Kodaira M, et al. CIC-rearranged sarcomas: a study of 20 cases and comparisons with Ewing sarcomas. Am J Surg Pathol 2016;40:313-323.

11 Somers GR, Shago M, Zielenska M, et al. Primary subcutaneous primitive neuroectodermal tumor with aggressive behavior and an unusual karyotype: case report. Pediatr Dev Pathol 2004;7:538-545.

12 Fletcher CDM, Unni KK, Mertens F, et al. World Health Organization Classification of Tumours: Pathology and Genetics of Tumours of Soft Tissue and Bone. IARC Press: Lyon, France, 2012.

13 Sankar S, Lessnick SL. Promiscuous partnerships in Ewing's sarcoma. Cancer Genet 2011;204:351-365.

14 Rakheja D, Goldman S, Wilson KS, et al. Translocation $(4 ; 19)(q 35 ; q 13.1)$-associated primitive round cell sarcoma: report of a case and review of the literature. Pediatr Dev Pathol 2008;11:239-244. 